\title{
Multi-module Singular Value Decomposition for Face Recognition
}

\author{
Bhanu Prasad $^{1}$, Sharad Kulkarini ${ }^{2}$ \\ 1. BhanuPrasad, M.Tech, SKTRMCE, \\ ${ }^{2}$ SharadKulkarini, M.Tech, Head of the Department(ECE), SKTRMCE,
}

\begin{abstract}
The paper introduces a face recognition method using probabilistic subspaces analysis on multimodule singular value features of face images. Singular value vector of a face image is valid feature for identification. But the recognition rate is low when only one module singular value vector is used for face recognition. To improve the recognition rate, many sub-images are obtained when the face image is divided in different ways, with all singular values of each image used as a new sample vector of the face image. These multi-module singular value vectors include all features of a face image from local to the whole, so more discriminant information for face recognition is obtained. Subsequently, probabilistic subspaces analysis is used under these multi-module singular value vectors. The experimental results demonstrate that the method is obviously superior to corresponding algorithms and the recognition rate is respectively $97.5 \%$ and $99.5 \%$ in ORL and CAS-PEAL-R1 human face image databases.
\end{abstract}

Keywords: face recognition; probabilistic subspaces analysis; multi-module; singular value decomposition;

\section{Introduction}

In recent years, computer vision research has witnessed a growing interest in subspace analysis techniques. And the important problem is how to extract the valid discriminant features for face recognition. The Karhunen-Loève Transform (KLT) and Principal Component Analysis are examples of eigenvector-based reduction and features extraction. A maximum a posteriori (MAP) matching rule using a Bayesian similarity measure derived from dual probabilistic has been applied for face recognition in. These methods have the disadvantages of the recognition performance easily affected by the facial expressions, large pose and variable illumination. A number of improved methods have been proposed. For example, the method using combination of wavelet packets and PCA/LDA have improved the face recognition rate, and Gabor representation based on probabilistic subspaces analysis for face recognition have also gotten the improvement of the face recognition, and so on. Their success shows that the traditional approaches applied in the different feature spaces can get the better recognition performance. Singular value features of matrix are widely used in the data compression, signal processing and pattern analysis, because they have the following features: the good stability, the proportional and rotational invariant. The singular value features of face images are firstly applied to face recognition in. The research of showed enough information needed could not be gotten for face recognition by using only one module singular value decomposition of whole face and the more features must be extracted. The paper introduces the face recognition method based on multi-module singular value features and probabilistic subspaces analysis (MSVD+PSA). Firstly, the sub-images are obtain when the face image is divided in different ways, then all singular value of each sub-image is organized and used as the sample vector of the face image. Finally, the method of probabilistic subspaces analysis is applied to the all sample vectors of face images. The remainder of the paper is organized as follows: In Section 2, the idea of the proposed method is described. The experimental results are presented for the ORL and CASPEAL- R1 face image databases to demonstrate the effectiveness and robustness of our method in section 3.Finally, conclusions are presented in Section 4.

\section{A. Multi-module Singular Value Decomposition:}

Our Face Recognition Method

The singular value decomposition (SVD) is one of the most important tools of numerical signal processing. It is employed in a variety of signal processing application, such as spectrum analysis, filter design, system identification etc. In singular value decomposition it has several advantages of computational efficiency and robustness under noise conditions. Singular value decomposition of matrix $A(m \times n)$ can be written as follows:

$$
A=U \Lambda V T(1)
$$

Where $U$-The columns of $U$ are the eigenvectors of AAT.U is an $m \times m$ matrix containing an orthonormal basis of vectors for both the columns space and left null space of $A$. For orbit correction, the orbit vector will be 
expanded in terms of the basis vectors in $U$. In linear algebra, $U$ contains the left singular vectors of $A . \Lambda$ - The 'singular values' or 'principle gains' of $A$ lie on the diagonal of $A$ and are the square root of eigenvectors of both $A A T$ and $A T A$, that is, the eigenvector in $U$ and the eigenvector of $V$ share the same eigenvalues. $V$ - The rows of $V T$ (columns of $V$ ) are the eigenvectors of $A A T$. $\mathrm{s}$ an $n \times n$ matrix containing an orthonormal basis ofvectors for both the row space and right null space of $A$. The column vector of corrector magnets vector will be expanded in terms of the basis vectors in $V$. $V$ contains the right singular vectors of $A$. We introduce the method of multimodule singular value decomposition. The $i$-by- $i$ sub-matrices $p A$ (where $p$ is the total number of sub-matrices, and if $i \times i \times p \neq m \times n$, the face image matrix A is filled by zero to ensure the both sides of the equation are equal) are gained when the face image matrix $A$ is divided in different ways. The sub-matrices $p A$ of face image are shown in Figure 1 when the modules $p$ is 16.

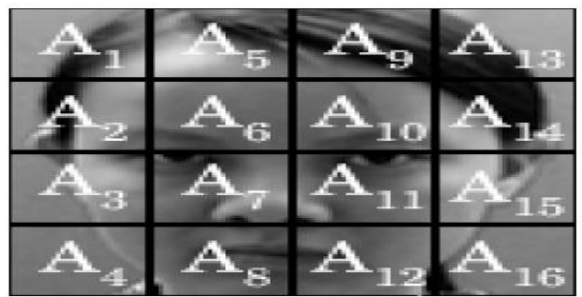

Figure 1. Example the face image is divided

The singular values $(1,2,,, p)$ of all sub-matrices $A_{k}$ are calculated by (1). Finally, the singular values $I_{k}$ are organized as follows:

$$
I=\left(I_{1}^{T}, I_{2}^{T}, \cdots, I_{p}^{T}\right)^{T}
$$

So, $I$ (where the size of $I$ is $i$-by- $p$ ) is used the sample vector of the matrix $A$. Because of the finite size of face image matrix $\mathrm{A}$, the divided number of face image matrix $\mathrm{A}$ is also finite.

\section{B. Probabilistic Subspace Analysis on the Singular Value of the Face Image:}

Based on the above results, we obtain a training set of vectors $\{I t\}$, where $I \in R N=i p$ and $t$ is the total number of training sample, by lexicographic ordering of the pixel elements of each matrix. The basis function for the KLT are obtained by solving the eigenvalue problem

$\Lambda=\Phi T \Sigma \Phi(3)$

Where $\Sigma$ is the covariance matrix, $\Phi$ is the eigenvector matrix of $\Sigma$, and $\Lambda$ is the corresponding diagonal matrix of eigenvalues. The unitary matrix $\Phi$ defines a coordinate transform (rotation) which de-correlates the data and makes explicit the invariant subspaces of the matrix operator $\Sigma$. In PCA, a partial KLT is performed to identify the largest eigenvalue eigenvectors and obtain a principal component feature vector $y=\Phi_{M}^{T} \tilde{I}$, where $\tilde{I}=I-\bar{I}$

mean normalized

vector

and $\phi_{M}^{T}$ is a sub-matrix of $\Phi$ containing the principal eigenvectors. PCA can be seen as a linear transformation $y=$ $\Gamma(I): R^{N} \rightarrow R^{M}$ which extracts a lower dimensional subspace of the KL basis corresponding to the first $\mathrm{M}$ largest eigenvalues. These principal components preserve the major linear correlations in the data and discard the minor ones. By ranking the eigenvectors of the KL expansion with respect to their eigenvalues and selecting the first M principal components, we form an orthogonal decomposition of vector space $R^{N}$ into two mutually exclusive

and complementary subspaces: the principal subspace (or feature space) $\bar{F}=\left\{\phi_{i}\right\}_{i=M+1}{ }^{N}$ containing the principal components and its orthogonal complement In practice there isalways a signal component in $F$ due to the minor statisticalvariability in the data or simply due to the observation noisewhich affects every element of I.The complete likelihood estimate can be written as theproduct of two independent marginal

$$
\begin{aligned}
\hat{P}(\Delta \mid \Omega) & =\left[\frac{\exp \left(-\frac{1}{2} \sum_{i=1}^{M} \frac{y_{i}^{2}}{\lambda_{i}}\right)}{(2 \pi)^{M / 2} \prod_{i=1}^{M} \lambda_{i}^{1 / 2}}\right] \cdot\left[\frac{\exp \left(-\frac{\varepsilon^{2}(\Delta)}{2 \rho}\right)}{(2 \pi \rho)^{(N-M) / 2}}\right] \\
& =P_{F}(\Delta \mid \Omega) \hat{P}_{\bar{F}}(\Delta \mid \Omega ; \rho),
\end{aligned}
$$


(4)Gaussian densitieswhere $P_{F}(\Delta \mid \Omega)$ is true marginal density in $\mathrm{F}, P_{F}(\Delta \mid \Omega p)$ is theestimated marginal density in the orthogonal complement $F, y_{i}$ are the principal components, and $\varepsilon^{2}(\Delta)$ is the PCAresidual (reconstruction error). The information-theoreticoptimal value for the density parameter $\rho$ is derived byminimizing the Kullback-Leibler (KL) divergence and isfound to be simply the average of the $F$ eigenvalues

$$
\rho=\frac{1}{N-M} \sum_{i=M+1}^{N} \lambda_{i}
$$

In their formulation, the above expression for $\rho$ is themaximum-likelihood solution of a latent variable model asopposed to the minimal-divergence solution.

\section{Classification}

We now consider the problem of characterizing the typeof differences which occur when matching two images in aface recognition task. We define two distinct and mutuallyexclusive classes: $\Omega_{\mathrm{I}}$ representing intrapersonal variationsbetween multiple images of the same individual (e.g., withdifferent expressions and lighting conditions), and $\Omega_{\mathrm{E}}$ representing extrapersonal variations which result whenmatching two different individuals. We will assume that bothclasses are Gaussian-distributed and seek to obtain estimatesof the likelihood functions $P\left(\Delta \mid \Omega_{I}\right)$ and $P\left(\Delta \mid \Omega_{E}\right)$ for a givenintensity difference $\Delta=I 1-I 2$.Given these likelihoods we can define the similarity score $S\left(I_{1}, I_{2}\right)$ between a pair of images directly in terms ofintrapersonal a posteriori probability as given by Bayes rules:

(6)Where the priors $P(\Omega)$ can be set to reflect specific operatingconditions (e.g., numbers of test images vs. the size of thedatabase) or other sources of a priori knowledge regarding thetwo images being matched. Additionally, this particularBayesian formulation casts the standard face recognition task(essentially an M-ary classification problem for M individuals)into a binary pattern classification problem with $\Omega_{I}$ and $\Omega_{\mathrm{E}}$.This much simpler problem is then solved using the maximuma posteriori (MAP) rule_-i.e., two images are determined tobelong to the same individual $P\left(\Delta \mid \Omega_{I}\right)>P\left(\Delta \mid \Omega_{E}\right)$, orequivalently, if $S\left(I_{1}, I_{2}\right)>1 / 2$.

$$
S=P\left(\Omega_{I} \mid \Delta\right)=\frac{P\left(\Delta \mid \Omega_{I}\right) P\left(\Omega_{I}\right)}{P\left(\Delta \mid \Omega_{I}\right) P\left(\Omega_{I}\right)+P\left(\Delta \mid \Omega_{E}\right) P\left(\Omega_{E}\right)}
$$

\section{Experimental Results}

\section{A. Experiment on the ORL Database}

The proposed method is tested on ORL face database.Database has more than one image of an individual's face withdifferent conditions (expression, illumination, etc). There areten different of each of 40 distinct subjects. Each image hasthe size of $112 \times 92$ pixels with 256 levels of gray. For somesubjects, the images were taken at different times, varying thelighting, facial expressions (open/close eye, smiling/notsmiling) and facial details (glasses / no glasses). All theimages were taken against a dark homogeneous backgroundwith the subjects in an upright, frontal position (with tolerancefor some side movement). The original pictures of $112 \times 92$ pixels have been resized to $64 \times 64$ so that the input space hasthe dimension of 4096 .In our experiment, as five images are chosen for training,the remaining images (unseen during training) are used fortesting. Thus, the total number of training samples and testingsamples is 200, respectively. Training set is composed asfollows: the first five images in per class for training, namedSet 1; the behind five images in per class for training, namedSet 2. The final result is the average of the Set1 and Set2.As the modules of the face image increase, the recognitionrate is improved in Figure 2. When the modules are equal to121, the recognition performance achieves the best result. Butthe modules are more than 121, there is lower recognition rate.This shows the recognition performance are greatly affectedby the divided modules of original image, and the way ofcombination of multi-module singular value features andprobabilistic subspaces analysis can be improvement of therecognition performance.For comparison purpose, we first implemented theMSVD+PSA method, the MSVD+LDA method, and theMSVD + PCA method. We use the modules of the face imageare equal to 121 in the experiment, and the recognition rate isthe average of Set1 and Set2. The comparative facerecognition performance of these three methods is shown inFigure 3. One can see from the figure that the MSVD+PSAmethod performs better than other methods. Especially, whenthe number of features is very little, 
the MSVD+PSA methodkeeps the high recognition rate.We compare the MSVD+PSA and PSA method (wherePSA method using the grey-value of original image). Theresult from Figure 4 show the MSVD+PSA method canachieve the recognition rate of $97.5 \%$, but the PSA methodonly can get the best recognition rate of 94\%. It also shows the MSVD+PSA method outperforms PSA.

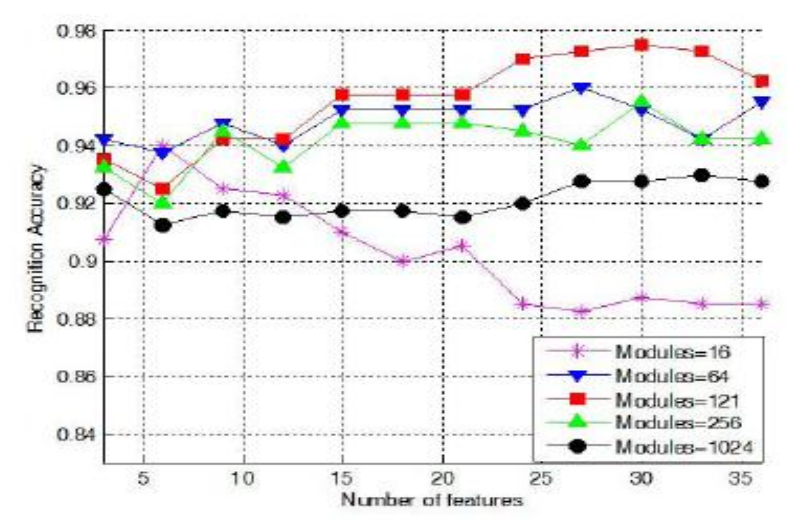

Figure 2. Recognition rate affected by different modules

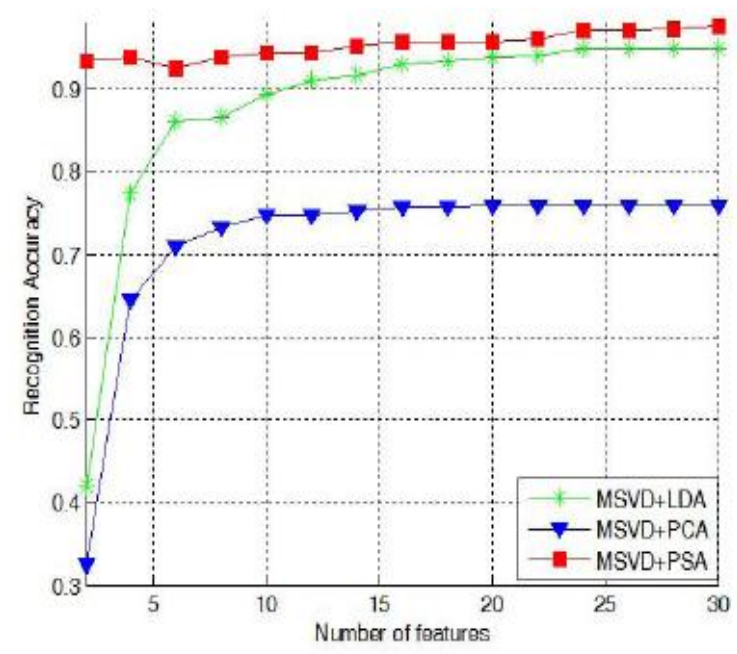

Figure 3.Comparative face recognition performance of the MSVD+PSAmethod, the MSVD+LDA method, and the MSVD + PCA method

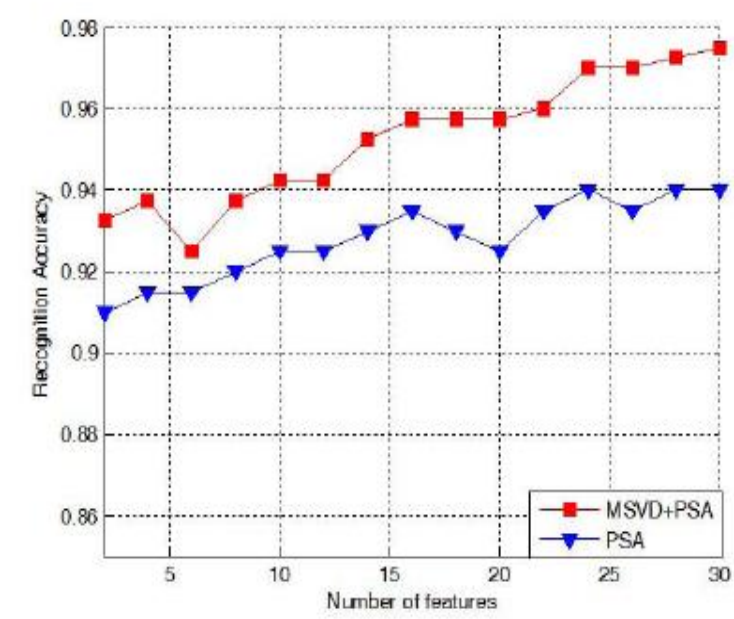

Figure 4. Comparative face recognition performance of the MSVD+PSA and PSA 


\section{B. Experiment on the CAS-PEAL-R1 Database}

CAS-PEAL-R1 Database is a large face database,containing 30863 images of the 1040 subjects. Theexperiments involve 1200 face images corresponding to 200subjects such that each subject has six images of size $360 \times 480$ with 256 gray scale levels. The face image is cropped tothe size of $64 \times 64$ to extract the facial region, which is furthernormalized to zero mean and unit variance. Figure 5 showssome example images used in our experiments that are alreadycropped to the size of $64 \times 64$. Note that they especiallydisplay different facial expressions.

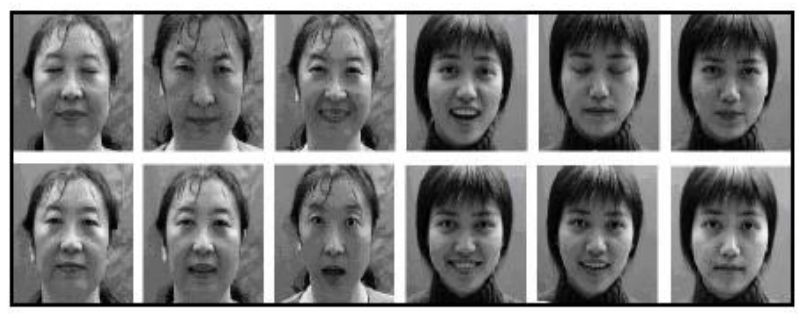

Figure 5.Example CAS-PEAL-R1 images used in our experiments.

\section{Conclusion}

We have introduced in the paper a novel multi-modulesingular value features and probabilistic subspace analysisclassification. The proposed method, which is robust tovariations in illumination and facial expression, applies thePSA method to the organized singular value features derivedfrom the multi-module of the face image. These multi-modulesingular value vectors include all features of a face image fromlocal to the whole, so more discriminant information forpattern recognition is obtained. So the recognition rate of theproposed method for face recognition is higher than those ofother methods in the image domain. The feasibility of ourmethod has been successfully tested on face recognition usingORL and 1200 CAS-PEAL-R1 frontal face imagescorresponding to 200 subjects, which were acquired undervariable illumination and facial expressions. The novel ourmethod achieves respectively $97.5 \%$ and $99.5 \%$ accuracy onface recognition of ORL and CAS-PEAL-R1 face databases.

\section{References:}

[1] Tian Yuan, Tan Tie-niu, Wang Yun-hong. "Do singular values containadequate information for face recognition", Pattern Recognition, vol.36,no.6, pp:649-655, 2003.

[2] Esther Annlin Kala James, Dr.S.Annaduai, etal. "Implementation ofincremental linear discriminant analysis using singular valuedecomposition for face recognition", ICAC,2009.

[3] BabackMoghaddam, Alex Pentland. "Probabilistic visual learning forobject represention", IEEE Trans. Pattern Analysis and MachineIntelligence, vol. 19, no. 7, pp. 696-710, July 2007.

[4] BabackMoghaddam. Watching Wahid and alexPenland. "Beyongeigenfaces: probabilistic matching for face recognition", proc. Int'l conf.Automatic Face and Gesture Recognition (FG'98),pp.30-35. Apr.1998.

[5] PN. Belhumeure, J.P. Hespanha, D.J. Kriengman, "Eigenfaces vs.fisherfaces: recognition using class spcific linear projection," IEEETrans. Pattern Analysis and Machine Intelligence, vol. 19, pp. 711-720,July 1997.

[6] W. .Gao, B.Cao, "The CAS-PEAL large-scale Chinese face database andbaseline evaluations," IEEE Transactions on Systems, Man, andCybernetics, vol. 38, pp. 149-161, January, 2008

\section{About the Authors}

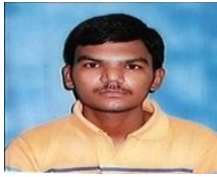

Bhanu Prasad .P,

M.Tech,

Sri KottamTulasi Reddy College Of Engineering \& Technology,

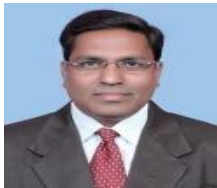

SharadKulkarni M.S(Ph.d)

Currently doing research on content based image retrieval in digital image processing stream.

Sri KottamTulasi Reddy College Of Engineering \& Technology, 